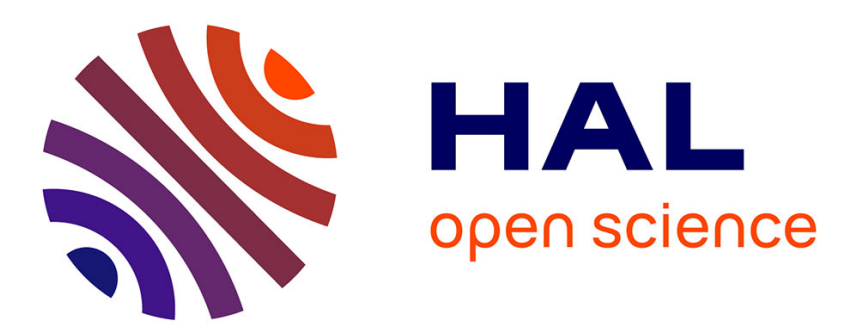

\title{
Ultra-narrow surface lattice resonances in plasmonic metamaterial arrays for biosensing applications
}

Artem Danilov, Gleb Tselikov, Fan Wu, Vasyl G. Kravets, Igor Ozerov, F. Bedu, Alexander N. Grigorenko, Andrei V. Kabashin

\section{To cite this version:}

Artem Danilov, Gleb Tselikov, Fan Wu, Vasyl G. Kravets, Igor Ozerov, et al.. Ultra-narrow surface lattice resonances in plasmonic metamaterial arrays for biosensing applications. Biosensors and Bioelectronics, 2018, 104, pp.102-112. 10.1016/j.bios.2017.12.001 . hal-01660871

\section{HAL Id: hal-01660871 https://hal.science/hal-01660871}

Submitted on 19 Dec 2018

HAL is a multi-disciplinary open access archive for the deposit and dissemination of scientific research documents, whether they are published or not. The documents may come from teaching and research institutions in France or abroad, or from public or private research centers.
L'archive ouverte pluridisciplinaire HAL, est destinée au dépôt et à la diffusion de documents scientifiques de niveau recherche, publiés ou non, émanant des établissements d'enseignement et de recherche français ou étrangers, des laboratoires publics ou privés. 


\title{
Ultra-narrow surface lattice resonances in plasmonic metamaterial arrays
}

\section{for biosensing applications}

\author{
Artem Danilov ${ }^{\mathrm{a}}$, Gleb Tselikov ${ }^{\mathrm{a}}$, Fan Wu $\mathrm{w}^{\mathrm{b}}$, Vasyl G. Kravets ${ }^{\mathrm{b}}$, Igor Ozerov ${ }^{\mathrm{c}}$, Frederic Bedu ${ }^{\mathrm{c}}$, \\ Alexander N. Grigorenko ${ }^{\mathrm{b}^{*}}$, Andrei V. Kabashin ${ }^{\mathrm{a}^{*}}$
}

a Aix-Marseille University, CNRS, LP3 UMR 7341, Campus de Luminy - Case 917, 13288, Marseille Cedex 9, France ${ }^{\text {bS }}$ chool of Physics and Astronomy, University of Manchester, Manchester M13 9PL, UK

${ }^{\mathrm{c}}$ Aix-Marseille University, CNRS, Centre Interdisciplinaire de Nanoscience de Marseille (CINaM) UMR 7325, Campus de Luminy, Case 913, 13288 Marseille, France

*Corresponding authors:

1) Tel.: +33-491829383, E-mail address: kabashin@1p3.univ-mrs.fr (Andrei V. Kabashin)

2) Tel.: +1-716-645-6800 x2099; E-mail address: Alexander.Grigorenko@manchester.ac.uk

*Equal contributions

\begin{abstract}
When excited over a periodic metamaterial lattice of gold nanoparticles $(\sim 100 \mathrm{~nm})$, localized plasmon resonances (LPR) can be coupled by a diffraction wave propagating along the array plane, which leads to a drastic narrowing of plasmon resonance lineshapes (down to a few nm full-widthat-half-maximum) and the generation of singularities of phase of reflected light. These phenomena look very promising for the improvement of performance of plasmonic biosensors, but conditions of implementation of such diffractively coupled plasmonic resonances, also referred to as plasmonic surface lattice resonances (PSLR), are not always compatible with biosensing arrangement implying the placement of the nanoparticles between a glass substrate and a sample medium (air, water). Here, we consider conditions of excitation and properties of PSLR over arrays of glass substrate-supported single and double Au nanoparticles $(\sim 100-200 \mathrm{~nm})$, arranged in a periodic metamaterial lattice, in
\end{abstract}


direct and Attenuated Total Reflection (ATR) geometries, and assess their sensitivities to variations of refractive index (RI) of the adjacent sample dielectric medium. First, we identify medium (PSLRair,

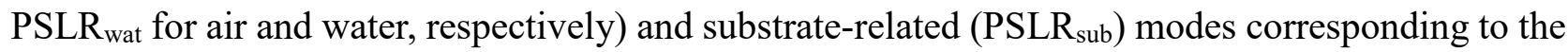
coupling of individual plasmon oscillations at medium- and substrate-related diffraction cut-off edges. We show that spectral sensitivity of medium-related modes to RI variations is determined by the lattice periodicity in both direct and ATR geometries ( $320 \mathrm{~nm}$ per RIU change in our case), while substrate-related mode demonstrates much lower sensitivity. We finally show that phase sensitivity of PSLR can exceed $10^{5}$ Deg. of phase shift per RIU change and thus outperform the relevant parameter for all other plasmonic sensor counterparts. Combining advantages of nanoscale architectures, including drastic concentration of electric field, possibility of manipulation at the nanoscale etc, and high phase and spectral sensitivities, PSLRs promise the advancement of current state-of-the-art plasmonic biosensing technology toward single molecule label-free detection.

Keywords: plasmonic surface lattice resonances, diffraction-coupled localized plasmon resonances, biosensor, attenuated total reflection, phase sensitivity, plasmonic metamaterials for biosensing

\section{Introduction}

For last decade plasmonic biosensing has become a technology of choice for label-free characterization of biomolecular binding interactions between a target analyte (antigens, DNA, ligand etc.) and its corresponding receptor (e.g. an antibody, DNA capture, protein etc.) immobilized on the gold surface (Liedberg et al., 1983, 1995, Schasfort et al., 2008, Homola, 2006). Plasmonic biosensors take advantage of a resonant dependence of conditions of plasmon excitation on refractive index (RI) of the medium contacting the plasmon-supporting metal. Conventional plasmonic biosensors employ the phenomenon of Surface Plasmon Resonance (SPR), which is associated with the excitation of surface plasmon polaritons over a thin $(\sim 50 \mathrm{~nm})$ gold film using Attenuated Reflection Geometry (Kretschmann-Raether arrangement). SPR effect leads to a dip in the reflected 
intensity at a defined combination of the angle of incidence and the wavelength, whose values are resonantly dependent on the RI of a thin layer near gold (Liedberg et al, 1983). Any change of the thickness of biological film on gold due to mass accumulation is accompanied by a RI change, which can be monitored by following spectral (Zhang et al., 1988), angular (Liedberg et al., 1983) or phase (Kabashin et al., 1997, 1998, 1999) characteristics of reflected light. With the lower detection limit of $1 \mathrm{pg} / \mathrm{mm}^{2}$ of biomaterial accumulated on the biosensor surface, SPR biosensors are efficient for studies of many biomolecular interactions (Schasfort et al., 2008), but they are hardly compatible with current trends in biosensing focusing on the development of compact (Patskovsky et al., 2004, Pilarik et al., 2003, Nemova et al., 2007) and nanoscale (Anker et al., 2008, Kabashin et al., 2009, Sreekanth et al., 2016) transducer implementations.

A huge potential for the upgrade of plasmonic biosensing technology is now expected from localized plasmon resonances (LPRs) excited over nanoscale structures (Anker et al., 2008), and metamaterial arrays (Kabashin et al., 2009). Such biosensors are much better compatible with nanoscale biochemical architectures (beacons, hybrid structures etc.), as well as can offer novel functionalities such as size-based selectivity, possibility for a drastic localization and concentration of electric field to profit from SERS and other field-enhanced effects (Nie et al., 2008), resolution beyond the diffraction limit (Kawata et al., 2009), nanotweezing (Grigorenko et al., 2008), etc. Plasmonic Surface Lattice Resonances (PSLR) produced in conditions of diffraction coupling of LPR are of particular interest for biosensing applications due to their exceptional resonance quality (Grigorenko et al., 2008, Auguie et al., 2008, Chu et al., 2008). To excite PSLR, gold nanoparticles (nanodics, nanopillars etc.) having the size of about $100 \mathrm{~nm}$ are arranged in a 2D lattice in such a way that one of diffracted beams, appearing due to the periodicity of this structure, propagates over the array plane and can couple in far field localized plasmons over individual nanoparticles (Fig. 1a). Such a coupling leads to a drastic narrowing of resonances in reflected and transmitted light down to 2-3 nm full width at half maximum (FWHM). In addition, the diffraction coupling gives rise to vanishing of light intensity in resonances, leading to the generation of singularities of light phase 
(Kravets et al., 2010, 2013). When used as a signal parameter to monitor refractive index variations, such phase singularities can be used to lower the detection limit of label-free plasmonic biosensing schemes down to single molecule level (Kabashin et al., 1997, 2009; Huang et al., 2012).

However, the excitation of diffraction-coupled PSLR is critically dependent on refractive index of the media surrounding a nanoparticle array. When the array is illuminated under normal incidence of light, the excitation of PSLR typically requires uniform surrounding, i.e. the match of refractive index of the substrate and that of the medium contacting the particles (Auguie et al., 2010), although certain narrowing of resonances is possible via a proper choice of size and geometry of nanoparticles (Chu et al., 2008, Trackray et al., 2014). On the other hand, the excitation of PSLR becomes possible under oblique incidence of light on the array structure and the monitoring of optical parameters in reflected light (Kravets et al., 2008, 2010). In all cases, direct geometry of PSLR excitation is not fully compatible with biosensing experimental arrangement, as it implies light direction through a sample liquid leading to a dependence of PSLR parameters on bulk RI fluctuations inside the flow cell. Alternatively, PSLR can be excited in Attenuated Total Reflection (ATR) geometry (Kravets et al., 2010), but conditions of excitation and sensitivity of such resonances have not yet been systematically studied.

In this paper, we compare conditions of PSLR excitation in direct and ATR geometries and examine sensitivities of different PSLR modes under these conditions. Our analysis shows that PSLR can be excited in both geometries and exhibit distinct resonance features with the spectral width of a few nm FWHM and high resonance quality. We also show that the PSLR can provide spectral sensitivity correlating with the periodicity of the structures and exceptionally high phase sensitivity, which makes them very promising candidates for biosensing applications.

\section{Methodology}

\subsection{Sample preparation}

High-quality regular and homogenous square arrays of gold nanoparticles were produced by 
e-beam lithography (LEO-RAITH) on a clean microscopic glass substrate covered by a thin $\mathrm{Cr}$ sublayer. A double layered resist was used to improve lift-off ( $80 \mathrm{~nm}$ of $495 \mathrm{kD} P M M A$ cast from a $3 \mathrm{wt} \%$ solution in anisole for the bottom resist layer and $50 \mathrm{~nm}$ of $95 \mathrm{kD}$ PMMA cast from a $2 \mathrm{wt} \%$ solution in anisole for the top layer). After lithography we deposited $5 \mathrm{~nm}$ of $\mathrm{Cr}$ (to improve adhesion) and $80-90 \mathrm{~nm}$ Au by electron beam evaporation, which was followed by the lift-off procedure. The typical array size was $0.2 * 0.2 \mathrm{~mm}^{2}$. The samples on a clean glass substrate were obtained from the samples fabricated on a $5 \mathrm{~nm} C r$ sublayer in which the $C r$ sublayer has been wet-etched after the fabrication procedure. Typical Scanning Electron Microscopy images of single and double nanodot arrays are shown in Fig. 1(b,c), respectively.

\subsection{Optical measurement setup}

In our experiments, we used commercially available M-2000 ellipsometer system (Woollam Inc, USA), which makes possible independent sample rotation and displacement, relative to illumination and collection light paths, thus granting the possibility for precise alignment of focal plane with respect to the examined area on a sample. Xenon wide spectrum lamp was used as a light source. The sample was illuminated with weakly focused light (about $0.5 \mathrm{~mm}$ spot diameter). Device output data represented in standard for ellipsometry $\Psi-\Delta$ values so that $E_{p} / E_{s}=\tan (\Psi) \exp (i \Delta)$, where $E_{p}$ and $E_{s}$ are the reflected field amplitudes for the incident light $\mathrm{E}_{\mathrm{i}}$ of $\mathrm{p}$ and s polarizations and their values are $R_{p}=\left|E_{p} / E_{i}\right|$ and $R_{s}=\left|E_{s} / E_{i}\right|$ of $\mathrm{p}$ and s polarizations, respectively.

A homemade liquid flow cell was designed for sensitivity measurements. The cell contained a rubber O-ring with input and output holes fixed between two cover slides for direct illumination measurements and a cover slide with 45-45-90 deg. glass prism $(n=1.5)$ on the other side for attenuated total reflection (ATR) configuration. In ATR geometry, the light beam was passed through a prism and reflected from its opposite facet where nanoparticle arrays were deposited. 


\section{Results and discussions}

\subsection{Conditions of excitation and properties of PSLR in direct and ATR geometries}

Under normal light illumination PSLR can be normally excited only under symmetric environment surrounding nanoparticle metamaterial arrays (Auguie et al., 2008, 2010), which prevents the implementation of biosensing schemes as the substrate and tested media typically have essentially different RI $\left(n_{\text {sub }} \sim 1.5\right.$ compared to $n_{\text {wat }}=1.33$ and $\left.n_{\text {air }}=1\right)$. However, this problem can be solved, e.g., by employing oblique light incidence (Kravets et al., 2008, 2010). In this case, PSLR can be efficiently excited even under asymmetry of optical environment, but they split into two modes corresponding to the coupling of localized plasmons via diffracted waves propagating in the tested medium or the substrate (Fig. 1a). The reason of this splitting is straightforward. For any regular nanoparticle array, diffracted beam disappears when it crosses the boundary between ambient medium (air or water) and the substrate due to the impossibility of transition between media having essentially different light dispersion. As a result, the diffracted beam is cut-off at Rayleigh cut-off wavelength (diffraction edge). It is obvious that if a medium/substrate interface is present, there should be two cut-off wavelengths. One of them corresponds to the disappearance of the medium diffraction modes (when the diffraction wave crosses the sample boundary from air/water to the substrate) and other one is related to crossing the boundary in the opposite direction. Such two Rayleigh cut-off wavelengths for air/substrate interface can be presented as follows (Kravets et al., 2008):

$$
\lambda_{R}^{\text {air }}=a / m\left(n_{\text {air }} \pm \sin \theta\right), \lambda_{R}^{\text {sub }}=a / m\left(n_{\text {sub }} \pm \sin \theta\right)
$$

where $a$ is a period of the structure, $m$ is integer, $\theta$ is the angle of incidence, $n_{\text {sub }}$ is substrate RI. Similarly, for water/substrate interface Rayleigh cut-off wavelengths will be:

$$
\lambda_{R}^{w a t}=a / m\left(n_{w a t} \pm \sin \theta\right), \lambda_{R}^{s u b}=a / m\left(n_{\text {sub }} \pm \sin \theta\right)
$$

The effect of splitting of resonances can be easily seen in experiments. Fig. 2 and Fig. 3 present spectral dependencies of ellipsometric parameters $\Psi$ and $\Delta$ for light reflected from a 320-nm period metamaterial arrays: an array of single $163 \mathrm{~nm}$ gold particles (panels (a)) and an array of 
double 134-nm particles having $\sim 50 \mathrm{~nm}$ separation (panels (b)), contacting with air and water media, respectively. It is visible that the resonances are indeed split into medium- and substrate-related

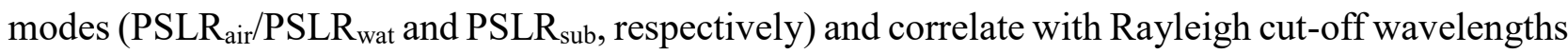
$\lambda_{R}^{\text {air }} / \lambda_{R}^{\text {wat }}$ and $\lambda_{R}^{s u b}$, which are indicated as vertical yellow lines on the spectra. It should be noted that for single nanoparticle arrays diffraction-coupled PSLRs match Rayleigh cut-off wavelengths (Fig. 2a, 3a), while for double nanoparticle arrays the position of PSLRs can remarkably shift from these wavelengths (Fig. 2b, 3b) due to near-field coupling in the nanoparticle dimer structure.

PSLR typically appear in reflection as narrow Fano-shape dips over a positive background spectrum (Kravets et al., 2008, 2010, Nikitin et al., 2013), presenting the combination of reflected and scattered light from glass-supported nanoparticle array system. However, when measured by ellipsometry and presented as a function of ellipsometric reflection $\Psi$, which describes a ratio of amplitudes of p- and s- components upon reflection $\tan \Psi=\frac{\left|R_{p}\right|}{\left|R_{s}\right|}$, the resonances can appear as both minima (PSLR ${ }_{\text {air }}$ for single and double nanoparticle arrays, Fig. 2, a,b) and maxima (PSLR $R_{\text {wat }}$ for

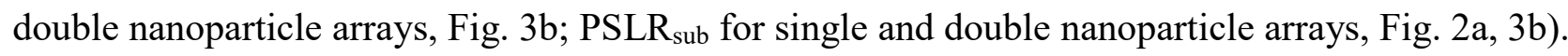
It is obvious that negative or positive polarity of $\mathrm{PSLR}_{\mathrm{S}}$ in $\Psi(\lambda)$ spectrum is determined whether resonant dips take place for $\mathrm{p}$ - or s-polarized component of light, respectively, or whether the substrate reflection adds or subtracted from the reflection produced by the array. In general, the width and shape of PSLR critically depend on a variety of factors, including nanoparticle size, geometry of unit cell (single or double particles), refractive index of the environment. In addition, the emergence of different modes and their intensity strongly depend on the angle of light incidence. Here, different structures appear to provide optimal resonance characteristics under different conditions. As an example, the single $163-\mathrm{nm}$ particle array provides a very distinct medium-related mode under its contact with air medium (Fig. 2a), while the same mode is almost absent in the case of its contact with water ambience (Fig. 3a). The substrate-related mode for this sample demonstrates the opposite tendency, as it appears to be much more pronounced in the case of water medium. As 
another example, the double 134-nm particle array provides much more pronounced medium-related mode under its contact with water medium (Fig. 2b, Fig. 3b), while the substrate-related mode shows the opposite tendency. As shown in figure insets presenting some resonances at a magnified scale, the width of PSLR is also strongly dependent on array parameters and range from a few nm FWHM to 20-25 nm FWHM. In all cases, the implementation of ultra-narrow and high quality resonances requires the optimization of array structures and their adaptation for concrete biosensing architectures (air, water). Fig. 2c, 3c show optical energy dispersion $E\left(k_{x}\right)$, which is constructed on the basis of experimental data (combinations of the resonance angle of incidence and wavelength). In fact, such dispersion summarizes conditions of excitation of medium- and substrate related modes in direct geometry for air and water environments, respectively. One can find that the dispersion curves $E\left(k_{x}\right)$ for both medium (PSLR ${ }_{\text {air }}$, PSLR $_{\text {wat }}$ ) and substrate (PSLR sub $_{\text {) }}$ modes have a characteristic descending course, which is related to a delocalized nature of diffraction-coupled plasmon surface lattice resonances (Odom et al., 2011, 2014).

One of critically important characteristics of resonant phenomena is related to light intensity in the very minimum of the resonance (light darkness). If the intensity approaches to zero, it can lead to singularities of phase of reflected light (Grigorenko et al., 1999), which can be used to much improve the sensitivity of plasmonic biosensing (Kabashin et al., 1997, 1998, 2009). As follows from Fig. 2 and 3, all PSLRs are accompanied by phase jumps, shown by dashed curves, in the very minima/maxima of the resonance. For example, the generation of air mode PSLR air for single 163 nm particle array (Fig. 2a) and water mode PSLR $_{\text {wat }}$ for double $134 \mathrm{~nm}$ particle array (Fig. 2b), manifested as a dip and peak in $\Psi(\lambda)$ dependence, respectively, is accompanied by extremely sharp phase jumps at the resonance points with a total phase variation around $180 \mathrm{Deg}$. Note that the generation of sharp phase jumps at resonant peaks and not dips does not contradict to our previous explanations of phase topological properties (Grigorenko et al., 1999, Kravets et al., 2014). Indeed, the appearance of PSLR as peaks in spectral dependence for $\Psi(\lambda)$ is explained by a particular way of presentation of ellipsometric data, as reflectivity of s-polarized component finds itself in the 
equation denominator. In normal reflection, this resonance should obviously appear as a minimum for s-polarized component.

Our experiments showed that all phenomena related to the excitation of PSLR over essentially asymmetric substrate medium interface can be efficiently reproduced in ATR geometry implying the illumination of the nanoparticle arrays from the glass substrate side. Furthermore, characteristics of such PSLR can be advantageous compared to the direct geometry. Fig. 4 and 5 show the excitation of PSLR in ATR geometry using the same arrays: single $163 \mathrm{~nm}$ particle array (panels a) and double $134 \mathrm{~nm}$ particle array (panels b), under their contact with air and water environments, respectively. Here, similarly to direct geometry, one can observe ultra-narrow PSLR modes near Rayleigh cut-off wavelengths, while the resonances equally appear as minima (e.g., PSLR $_{\text {air }}$ and PSLR wat $_{\text {for }}$ single $163 \mathrm{~nm}$ particle and double $134 \mathrm{~nm}$ particle arrays) or maxima (PSLR $_{\text {sub }}$ for the $163 \mathrm{~nm}$ particle array contacting air, and PSLR sub for the double $134 \mathrm{~nm}$ particle array contacting water). As in the case of direct geometry, PSLRs are generated exactly at Rayleigh cut-off wavelengths for single nanoparticle arrays (Fig. 4a, 5a), while for double nanoparticle arrays the position of PSLR can be much shifted (Fig. 5b). The width and quality of resonances also critically depends on nanoparticle size, geometry of unit cell (single or double particles), refractive index of the environment. As an example, the array of single 163-nm particles demonstrates a prominent medium related mode under its contact with air (Fig. 4a), while this resonance is very weak under the contact of this structure with water ambience (Fig. 5a). Based on statistics of PSLR excitation in ATR geometry using various samples, we can conclude that single nanoparticle arrays demonstrate much better medium resonances under their contact with air ambience, while double nanoparticle arrays can provide high quality medium-related resonances for both air and water ambiences. As shown in insets to Fig. 4 and Fig. 5, the widths of PSLR in the ATR geometry are in average smaller compared with this parameter in direct geometry and can range from a few nm to 15-20 nm FWHM. Similarly to the case of direct geometry, the generation of PSLRs is accompanied by sharp phase features and the sharpness of phase jump is determined by the depth of resonances. 
As an example, very sharp phase features with total phase variation close to $180 \mathrm{Deg}$. are observed for medium related mode PSLR air using single $163 \mathrm{~nm}$ particle array (inset of Fig. 4a) and for PSLR air, PSLR $_{\text {wat }}$ using double $134 \mathrm{~nm}$ particle arrays (insets of Fig. 4b, Fig. 5b). As shown in Fig. Fig. 4c, 5c, optical dispersion $E\left(k_{x}\right)$ curves for medium (PSLR ${ }_{\text {air }}$ and PSLR $\left.{ }_{\text {wat }}\right)$ and substrate $\left(\right.$ PSLR $\left._{\text {sub }}\right)$ modes demonstrate the same descending trend as it was observed in direct geometry. It should be noted that arrays of single 190-nm particles reveal a negative dispersion for PSLR air mode, which could be related to too large size of gold nanoparticles and possible involvement of complex plasmon modes (e.g., quadruple ones).

Thus, ATR geometry makes possible the excitation of ultra-narrow diffraction-coupled plasmonic surface lattice resonances over arrays of gold nanodots contacting both air and water ambiences, while characteristics of these resonances can be optimized by a proper selection of array parameters. Our analysis shows that PSLR excited under ATR can be even narrower than relevant resonances under direct geometry.

\subsection{Sensitivity of PSLR to local environment}

Ultimate biosensing experiment implies the functionalization of the plasmonic nanoparticles and immobilization of a sensor recognition element on them. A target analyte (affinity partner) comes from aqueous ambience and is supposed to bind to the recognition element leading to an increase of refractive index of a thin layer around the nanoparticles, while a relevant change of conditions of PSLR excitation (spectral, phase) enables one to monitor the RI increase caused by the biological interaction. In this case, the resulting response of biosensor system is determined by both optical sensitivity of the plasmonic transducer to RI variations and the efficiency of the recognition element. Since the main objective of this work is related to the development of optical transducer based on PSLR, we use a physical model to simulate RI changes in order to simplify the analysis and avoid artificial facts related to the performance of the recognition element. To simulate the increase of RI of water ambience, aqueous solutions of ethanol of different concentrations were pumped through a 
flow cell by a peristaltic pump and brought into contact with the nanoparticle arrays, while PSLR were excited in both direct and ATR geometries. A block containing the metamaterial slide with nanoparticle arrays and the flow cell was put onto the ellipsometer platform and examined.

Figure $6 \mathrm{a}$ shows spectral shifts of resonances due to changes of refractive index of the aqueous medium caused by the addition of different concentration of ethanol to water for an array of single 190-nm nanoparticles. It is visible that an increase of refractive index (RI) due to the ethanol addition leads to a red shift of PSLR and this shift is nearly linear. However, sensitivities of medium and substrate-related modes appear to be quite different. Here, the slope of the linear dependence for

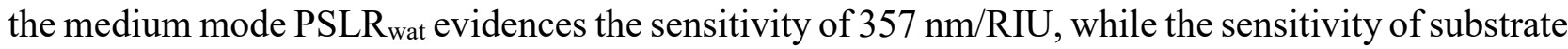
mode is much lower $(200 \mathrm{~nm} / \mathrm{RIU})$. Statistical analysis of sensitivities of medium and substrate modes for various samples shows that this parameter does not depend whether single or double nanoparticle arrays are used and is in the range of 310-360 nm/RIU and 190-200 nm/RIU for PSLR wat $_{\text {. }}$ and PSLR sub, respectively. In general, sensitivity of medium modes PSLR $_{\text {wat }}$ for different samples strongly correlates with the period of structures $(320 \mathrm{~nm})$, similarly to other transducers based on diffraction phenomena such as e.g., surface plasmon polaritons over nanohole thin film arrays (Brolo et al., 2004), while sensitivity of the substrate mode PSLR wat does not show any correlation with the array period and is much lower.

A more complicated situation takes place for sensitivities of medium and substrates modes in ATR geometry. Here, the value of sensitivity of the medium mode starts to depend whether its excitation takes place before or after the angle of total internal reflection (TIR) for glass/water interface $\left(\sim 63^{\circ}\right.$ Deg.) Indeed, as follows from Fig. 6 b, sensitivity of PSLR wat $_{\text {before the TIR angle }}$ ( $\sim 46^{\circ}$ Deg.) is also of the order of the period of the structure ( $305 \mathrm{~nm} / \mathrm{RIU}$ for double $134 \mathrm{~nm}$ particle arrays), while for angles of incidence after TIR ( $73^{\circ}$ Deg.) this parameter can exceed $400 \mathrm{~nm} / \mathrm{RIU}$. Note that such a difference of sensitivities was recorded for a largely dominating majority of samples of single and double nanodot arrays, independently of the geometry of the unit cell. Here, the samples typically demonstrate sensitivity in the range of $300-340 \mathrm{~nm} / \mathrm{RIU}$ before the TIR angle and improved 
sensitivity of 400-420 nm/RIU after the TIR angle. On the other hand, as follows from $6 \mathrm{~b}$, substraterelated mode appears to be weakly sensitive to variations of RI (50-55 nm/RIU) independently of angle of incidence (before or after TIR).

It is clear that sensitivities of PSLR modes can be obtained within diffraction theory, but a detailed consideration of the phenomenon should take into account not only the dependence of diffraction edge position on RI of the environment, but also accompanying effects such as a certain mismatch of actual PSLR positions with that of the Rayleigh cut-off wavelengths and evanescent near-fields in the case of ATR geometry. However, in the first approximation, this sensitivity should correlate with the sensitivity of the diffraction edge. The position of this Rayleigh cut-off wavelength for water and substrate modes in direct geometry can be derived as follows:

$$
\begin{gathered}
\pm \lambda_{R_{-} \text {Dir }}^{\text {Wat }}=a n_{\text {Wat }}(\sin \theta \pm 1) \\
\pm \lambda_{R_{-} \text {Dir }}^{\text {Sub }}=a\left(n_{\text {wat }} \sin \theta \pm n_{\text {sub }}\right)
\end{gathered}
$$

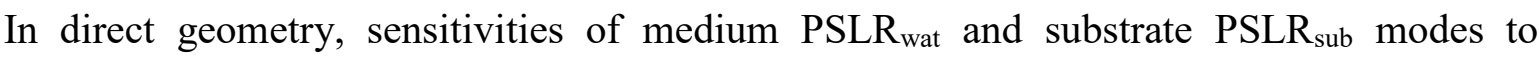
variations of refractive index of the aqueous medium can be expressed as follows:

$$
\begin{aligned}
& S_{\text {dir }}\left(P S L R_{\text {wat }}\right)=\frac{\partial \lambda_{R_{-} \text {Dir }}^{\text {wat }}}{\partial n}=\frac{\partial}{\partial n}[a n(\sin \theta \pm 1)]=a\left(\sin \theta \pm 1+\frac{\delta \sin \theta}{\delta n}\right) \\
& S_{\text {dir }}\left(P S L R_{\text {sub }}\right)=\frac{\partial \lambda_{R_{D} \text { Dir }}^{\text {Sub }}}{\partial n}=\frac{\partial}{\partial n}\left[a\left(n \sin \theta \pm n_{\text {sub }}\right)\right]=a\left(\sin \theta+n \frac{\delta \sin \theta}{\delta n}\right)
\end{aligned}
$$

where $n$ is refractive index of ethanol/water mixture. Since the angle of light incidence at water/substrate interface $\theta$ can be derived through the angle of light incidence $\alpha$ at the air/water interface (this interface is located at the flow cell entrance window) by the Snell's law $n_{\text {air }} \cdot \sin \alpha=$ $n \cdot \sin \theta$, two latter equations can be rewritten as:

$$
\begin{gathered}
S_{\text {dir }}\left(P S L R_{\text {wat }}\right)=a\left(\sin \theta \pm 1-\frac{n n_{\text {air }} \sin \alpha}{n^{2}}\right)=a(\sin \theta \pm 1-\sin \theta)= \pm a \\
S_{\text {dir }}\left(P S L R_{\text {sub }}\right)=a\left(\sin \theta-\frac{n n_{\text {air }} \sin \alpha}{n^{2}}\right)=a(\sin \theta-\sin \theta)=0
\end{gathered}
$$

Thus, according to simplified diffraction theory, sensitivity of the medium mode (PSLR wat $_{\text {) }}$ to RI in direct geometry is supposed to be of the order of the period of the structure (a nm/RIU), 
while the substrate mode should be insensitive to RI variations.

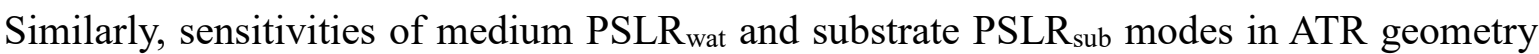
are mainly determined by positions of corresponding Rayleigh cut-off wavelength:

$$
\begin{gathered}
\pm \lambda_{R_{-} A T R}^{W a t}=a\left(n_{\text {sub }} \sin \theta \pm n_{\text {Wat }}\right) \\
\pm \lambda_{R_{-} A T R}^{\text {Sub }}=a n_{\text {sub }}(\sin \theta \pm 1)
\end{gathered}
$$

Therefore, these sensitivities can be expressed as follows:

$$
\begin{gathered}
S_{A T R}\left(P S L R_{w a t}\right)=\frac{\partial \lambda_{R A T R}^{w a t}}{\partial n}=\frac{\partial}{\partial n}\left[a\left(n_{s u b} \sin \theta \pm n\right)\right]= \pm a \\
S_{A T R}\left(P S L R_{\text {sub }}\right)=\frac{\partial \lambda_{R}^{S u b} A R R}{\partial n}=\frac{\partial}{\partial n}\left[a n_{s u b}(\sin \theta \pm 1)\right]=0
\end{gathered}
$$

Here, again, sensitivity of the medium mode (PSLR ${ }_{\text {wat }}$ ) in ATR appears to be linked to the period of the structure (a nm/RIU), while the substrate mode should be insensitive to these variations.

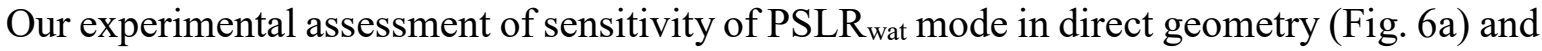
ATR geometry before TIR angle (Fig. 6b) confirm these theoretical predictions, as these sensitivities are in the range of $310-360 \mathrm{~nm} / \mathrm{RIU}$ and $340-340 \mathrm{~nm} / \mathrm{RIU}$, respectively. However, a higher sensitivity of this mode in ATR geometry (400-420 nm/RIU) under angles exceeding the TIR angle is a pleasant surprise taking into account that this sensitivity does not follow from diffraction theory considerations. We believe that this effect is related to the involvement of evanescent wave under TIR, which can additionally react on RI variations. Another interesting issue is related to the observation of some dependence of substrate related modes PSLR sub $_{\text {on }}$ RIU variations for direct and ATR geometries (190-200 nm/RIU and 55-55 nm/RIU, respectively), although diffraction theory can not predict these dependencies. We believe that such a discrepancy of experiment and theory is related to too simplified model used in our calculations. In this model, we considered the sensitivity of the diffraction edge, which is not far from actual resonances for oblique angles, but still does not match them. Nevertheless, even if the deviation from Rayleigh wavelength is small, it changes fast with n, angle, etc, as the observed PSLR are very dispersive (Fig. 2c, 3c, 4c, 5c), providing non-zero sensitivities for substrate modes. In addition, the proposed theory does not take into account the 
excitation of evanescent wave under illumination of arrays at angles larger than $63^{\circ}$ Deg. using ATR geometry, which can explain unexpectedly high sensitivity of medium mode under these conditions (Fig. 6 b).

Let us now consider phase sensitivity of plasmonic surface lattice resonances. It should be noted that this parameter does not directly correlate with the spectral sensitivity. Indeed, as we

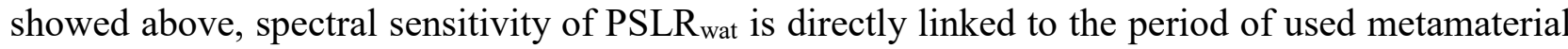
arrays and does not depend on width of depth of generated resonances. On the other hand, phase sensitivity is independent of the period of the arrays, but is determined by the sharpness of phase jump, which in turn is critically dependent on the depth of resonances ("light darkness" in the resonant minimum) (Grigorenko et al., 1999). As an example, one can expect high phase sensitivity for double 134-nm particle arrays, which provide very deep resonances under ATR geometry leading to extreme phase features (Fig. $4 \mathrm{~b}$ and $5 \mathrm{~b}$, respectively).

As shown in Figure $6 c$, the replacement of $80 \%$ solution of ethanol ( $80 \%$ ethanol / $20 \%$ water) by $100 \%$ ethanol solution, corresponding to a change of RI by $4.4 \cdot 10^{-3}$ RIU, leads to a dramatic shift of phase curve (Fig. 6c) and a huge ( $\sim 205^{\circ}$ Deg. $)$ change of phase at the optimal wavelength of about $899 \mathrm{~nm}$ (inset to Fig. 6c). Based on this data, we can now estimate phase sensitivity, which appears to be equal to $5.7 \cdot 10^{4}$ Deg. of phase per RIU. Taking into account the level of noises of phasesensitive detection schemes, we can then estimate the low detection limit (LOD), which characterizes minimal variation of RI detectable by an optical transducer. It is clear that LOD should be very high for ellipsometry, as commercial ellipsometers are typically designed for optical characterization of films and not for sensing tests, and thus have a high level of noises. However, as shown in our previous study (Kabashin et al., 2009), resolution of phase measurements using an advanced photoacoustic modulator-based scheme of phase detection and a thermally stabilized flow cell system can be better than $5 \times 10^{-3}$ Deg. Under this phase resolution, projected LOD is estimated to be lower than $8 \cdot 10^{-8} \mathrm{RIU}$, which is better than in commercial amplitude-sensitive SPR units by almost 2 orders of magnitude (Lindberg et al., 1995). Notice that the minimal variation of RI that could be 
introduced into our system using calibrated ethanol-water mixtures was of the order of $4 \cdot 10^{-3}$ RIU. Smaller variations of RI could not be resolved due to too high level of noises, conditioned by liquid pumping system and flow cell. As shown in Fig. 6c, such a variation of RI already exceeded the dynamic range of phase measurements, which inevitably lowered our estimation of phase sensitivity. In our previous study (Kravets et al., 2010), we assessed phase sensitivity of similar nanoparticle array metamaterials using a gas model, which made possible the introduction of much small variations of RI of the medium by employing a calibrated mixture of gases with known difference of RI (Ar and $\mathrm{N}_{2}$ ). In this case, the system was in the optimal dynamic range for the whole set of phase measurements, while the recorded sensitivity exceeded $10^{6}$ Deg. of phase shift per RIU, giving access to ultrasensitive measurements of RI with the detection limit lower than $10^{-9}$ RIU.

Thus, ultra-narrow PSLRs over ordered nanodot array metamaterials can exhibit fairly good sensitivity (300-400 nm/RIU), conditioned by the periodicity of used structures and extremely high phase sensitivity, conditioned by a very low light intensity in the resonance minimum (nearly complete light darkness). It is clear that a much reduced spectral width of PSLRs (2-20 nm FWHM) compared to LPR (80-100 nm FWHM) and SPR (50 nm FWHM) can be used to improve the precision of biosensing tests using plasmonic biosensors. To take into account the sharpness of the resonance and thus examine system efficiency to sensitively measure small wavelength changes, one normally uses a characteristic "Figure of Merit" (FOM) parameter (Mayer et al., 2011): $\mathrm{FOM}=(\Delta \lambda / \Delta n)(1 / \Delta \omega)$, where $\Delta \omega$ is the width of resonance at FWHM and $\Delta \lambda$ is the resonance shift for a $\Delta n$ refractive-index change. The essence of FOM is straightforward: it adequately quantifies the sensing potential of plasmonic modes in configurations similar to those used in commercial instruments. Typical FOMs do not exceed 8 and 23 for the sensors based on LPR (Anker et al., 2008) and SPR (Zhang et al., 1988), respectively. The employment of PSLRs enables one to increase these parameters up to 10,100 and more. In particular, in our experiments FOM reached 183.8, which is in a good agreement with our earlier works there it reached $\sim 200-250$ (Kravets et al., 2010). As another illustration, it was recently shown (Offermans et al., 2011) that PSLRs in ordered 
metamaterial arrays of gold nanoparticles can offer a sensing performance that is at least an order of magnitude improvement over uncoupled LPRs associated with disordered particle arrays. Other studies experimentally demonstrated a high-performance RI-sensitive sensor based on PSLR, while its FOM reached 38 normal incidence (Li et al., 2015; Shen et al., 2015). However, the main advantage offered by PSLR is related to their phase sensitivity, which can be extremely high (over $10^{6}$ Deg. of phase shift per RIU change). In general, the combination of ultrahigh point sensitivity (provided by phase interrogation) and fairly high sensitivity for a wider dynamic range of measurements (provided by spectral interrogation) looks like a very attractive basis for the development of versatile biosensing platform based on PSLR for characterization of biomolecular interactions.

The reported spectral sensitivity of PSLRs $(300-400 \mathrm{~nm} / \mathrm{RIU})$ is comparable with the best values of sensitivity reported for alternative nanoperiodic plasmonic transducers, including uncoupled plasmonic arrays (200-300 nm/RIU) (Anker et al, 2008) and nanohole arrays (300-400 $\mathrm{nm} / \mathrm{RIU}$ ) (Brolo et al., 2004; Im et al., 2011), but almost one order of magnitude lower than in the case of SPR using Kretschmann-Raether prism arrangement ( 2000-4000 nm/RIU) (Zhang et al., 1988). However, this tendency is not valid for phase sensitivities, which depend on "light darkness" in resonances and manifest themselves quite differently. As an example, even after a rigorous optimization and the thickness of thin Au films, SPR does not render possible intensities lower than $\sim 5 \%$ in the resonance minimum compared to off-resonance conditions (Kabashin et al., 2009). As a result, the jump of phase of the light is considerably smoothed out, such a phase smoothing effect limits phase sensitivity of this approach by $(5-10) \cdot 10^{4}$ Deg. of phase per RIU. The employment of diffractively coupled PSLR enables one to achieve extremely low light intensities (down to $10^{-6} \%$ ) (Kravets et al., 2008, 2010, 2013) and thus obtain extreme phase sensitivities exceeding $10^{6}$ Deg. per RIU change. Thus, despite a considerable handicap of PSLR in terms of amplitude sensitivity, the use of phase sensitivity completely compensates for this. To the best of our knowledge, PSLRs outperform all plasmonic counterparts in terms of phase sensitivity. 


\section{CONCLUSIONS}

We established conditions of excitation and properties of diffraction-coupled plasmonic surface lattice resonances over metamaterial arrays of single and double Au nanoparticles in direct and ATR geometries. We first identified the possibility for excitation of medium and substrate-related modes, corresponding to the coupling of individual plasmon oscillations at medium- and substraterelated diffraction cut-off edges. We also determined that spectral sensitivity of medium modes is conditioned by the periodicity of used structures and is of the order of $300-400 \mathrm{~nm} / \mathrm{RIU}$, while substrate-related modes have much lower sensitivity. Finally, we found that PSLR can exhibit extremely high phase sensitivity due to a very low light intensity in the resonance minimum (nearly complete light darkness). Combining ultrahigh point sensitivity (provided by phase interrogation) and fairly high sensitivity for a wider dynamic range of measurements (provided by spectral interrogation) and a series of advantages of nanoscale metamaterial architecture, the proposed sensor transducer based on PSLR can serve as a basis for the development of versatile platform for ultrasensitive characterization of biomolecular interactions.

\section{ACKNOWLEDGEMENTS}

AD, GT, AVK acknowledge the support from A*MIDEX project (No. ANR-11-IDEX-000102) funded by the "Investissements d'Avenir" French Government program, managed by the ANR, and LASERNANOCANCER and GRAVITY projects of ITMO “Plan Cancer 2014-2019” INSERM program. rANG acknowledges the support from the European Union's Horizon 2020 research and innovation programme under grant agreement No. 696656 "GrapheneCore1", the EPSRC (EP/K011022/1) and SONY research agreement. 


\section{References}

Anker, J.N., Hall, W.P., Lyandres, O., Shah, N.C., Zhao, J., Van Duyne, R.P., 2008. Biosensing with plasmonic nanosensors. Nat. Mater. 7, 442-453. doi:10.1038/nmat2162

Auguie, B., Barnes, W.L., 2008. Collective resonances in gold nanoparticle arrays. Phys. Rev. Lett. 101, 1-4. doi:10.1103/PhysRevLett.101.143902

Brolo, A.G., Gordon, R., Leathem, B., Kavanagh, K.L., 2004. Surface plasmon sensor based on the enhanced light transmission through arrays of nanoholes in gold films. Langmuir 20, 48134815. doi:10.1021/la0493621

Chu, Y., Schonbrun, E., Yang, T., Crozier, K.B., 2008. Experimental observation of narrow surface plasmon resonances in gold nanoparticle arrays. Appl. Phys. Lett. 93, 2006-2009. doi:10.1063/1.3012365

Grigorenko, A.N., Roberts, N.W., Dickinson, M.R., Zhang, Y., 2008. Nanometric optical tweezers based on nanostructured substrates. Nat. Photonics 2, 365-370. doi:10.1038/nphoton.2008.78

Grigorenko, A.N., Nikitin, P.I., Kabashin, A.V, 1999. Phase jumps and interferometric surface plasmon resonance imaging. Appl. Phys. Lett. 75, 3917-3919. doi:10.1063/1.125493

Homola, J., 2006. Surface Plasmon Resonance Based Sensors. Springer.

Huang, Y.H., Ho, H.P., Kong, S.K., Kabashin, A.V., 2012. Phase-sensitive surface plasmon resonance biosensors: Methodology, instrumentation and applications. Ann. Phys. 524, 637-662. doi:10.1002/andp.201200203

Im, H., Lee, S.H., Wittenberg, N.J., Johnson, T.W., Lindquist, N.C., Nagpal, P., Norris, D.J., Oh, S.H., 2011. Template-stripped smooth Ag nanohole arrays with silica shells for surface plasmon resonance biosensing. ACS Nano 5, 6244-6253. doi:10.1021/nn202013v

Kabashin, A.V., Kochergin, V.E., Beloglazov, A.A., Nikitin, P.I., 1998. Phase-polarisation contrast for surface plasmon resonance biosensors1This paper was presented at the Fifth World Congress on Biosensors, Berlin, Germany, 3-5 June 1998.1. Biosens. Bioelectron. 13, 1263-1269. doi:10.1016/S0956-5663(98)00088-8

Kabashin, A.V., Nikitin, P.I., 1997. Interferometer based on a surface-plasmon resonance for sensor applications. Quantum Electron. 27, 653-655. doi:10.1070/QE1997v027n07ABEH001013

Kabashin, A. V., Kochergin, V.E., Nikitin, P.I., 1999. Surface plasmon resonance bio- and chemical sensors with phase-polarisation contrast. Sensors Actuators, B Chem. 54, 51-56. doi:10.1016/S0925-4005(98)00326-8

Kabashin, A.V, Evans, P., Pastkovsky, S., Hendren, W., Wurtz, G.A., Atkinson, R., Pollard, R., Podolskiy, V.A., Zayats, A. V, 2009. Plasmonic nanorod metamaterials for biosensing. Nat. Mater. 8, 867-871. doi:10.1038/nmat2546

Kabashin, A.V, Patskovsky, S., Grigorenko, A.N., Méditerranée, U. De, 2009. Phase and amplitude sensitivities in surface plasmon resonance bio and chemical sensing 17, 151-161.

Kawata, S., Inouye, Y., Verma, P., 2009. Plasmonics for near-field nano-imaging and superlensing. 
Nat. Photonics 3, 388-394. doi:10.1038/nphoton.2009.111

Kravets, V.G., Schedin, F., Grigorenko, A.N., 2008. Extremely narrow plasmon resonances based on diffraction coupling of localized plasmons in arrays of metallic nanoparticles. Phys. Rev. Lett. 101, 1-4. doi:10.1103/PhysRevLett.101.087403

Kravets, V.G., Schedin, F., Jalil, R., Britnell, L., Gorbachev, R. V, Ansell, D., Thackray, B., Novoselov, K.S., Geim, A.K., Kabashin, A.V, Grigorenko, A.N., 2013. Singular phase nano-optics in plasmonic metamaterials for label-free single-molecule detection. Nat. Mater. 12, 304-9. doi:10.1038/nmat3537

Kravets, V.G., Schedin, F., Kabashin, A.V, Grigorenko, A.N., 2010. Sensitivity of collective plasmon modes of gold nanoresonators to local environment. Opt. Lett. 35, 956-958. doi:10.1364/OL.35.000956

Liedberg, B., Nylander, C., Lundström, I., 1995. Biosensing with surface plasmon resonance - how it all started. Biosens. Bioelectron. 10. doi:10.1016/0956-5663(95)96965-2

Liedberg, B., Nylander, C., Lunström, I., 1983. Surface plasmon resonance for gas detection and biosensing. Sensors and Actuators 4, 299-304. doi:10.1016/0250-6874(83)85036-7

Mayer, K.M., Hafner, J.H., Antigen, A.À., 2011. Localized surface plasmon resonance sensors. Chem. Rev. 111, 3828-3857. doi:10.1021/cr100313v

Nemova, G., Kabashin, A.V., Kashyap. R., 2008. Surface Plasmon Polariton Mach-Zehnder refractive index sensor. J. Am. Opt. Soc. B, 25, 1673-1677. doi:10.1364/josab.25.001673

Nie, S. and Emory, S.-R., 1997. Probing Single Molecules and Single Nanoparticles by SurfaceEnhanced Raman Scattering. Science. 275, 1102-1106. doi:10.1126/science.275.5303.1102

Nikitin, A.G., Kabashin, A.V., Dallaporta, H., 2012. Plasmonic resonances in diffractive arrays of gold nanoantennas: Near and far field effects. Opt. Express 20, 27941-27952. doi:10.1364/oe.20.027941

Offermans, P., Schaafsma, M.C., Rodriguez, S.R.K., Zhang, Y., Crego-Calama, M., Brongersma, S.H., Gómez Rivas, J., 2011. Universal scaling of the figure of merit of plasmonic sensors. ACS Nano 5, 5151-5157. doi:10.1021/nn201227b

Patskovsky, S., Kabashin, A.V., Meunier, M., Luong, J.H.T., 2003. Multi-layer Si-Based Surface Plasmon Resonance Structure for Absorption Sensing. Anal. Lett. 36, 3261-3270. doi:10.1081/AL-120026570

Piliarik, M., Homola, J., Maníková, Z., Čtyroký, J., 2003. Surface plasmon resonance sensor based on a single-mode polarization-maintaining optical fiber. Sensors Actuators, B Chem. 90, 236-242. doi:10.1016/S0925-4005(03)00034-0

Schasfoort, R.B.M., Tudos, A. J., 2008. Handbook of Surface Plasmon Resonance. Royal Society of Chemistry.

Sreekanth, K.V., Alapan, Y., ElKabbash, M., Ilker, E., Hinczewski, M., Gurkan, U.A., De Luca, A., Strangi, G., 2016. Extreme sensitivity biosensing platform based on hyperbolic metamaterials. Nat. Mater. 15, 4-11. doi:10.1038/nmat4609 
Thackray, B.D., Kravets, V.G., Schedin, F., Auton, G., Thomas, P.A., Grigorenko, A.N., 2014. Narrow Collective Plasmon Resonances in Nanostructure Arrays Observed at Normal Light Incidence for Simplified Sensing in Asymmetric Air and Water Environments. ACS Photonics 1, 1116-1126. doi:10.1021/ph5002186

Zhang, L.-M., Uttamchandani, D., 1988. Optical chemical sensing employing surface plasmon resonance. Electron. Lett. 24, 1469-1470. doi:10.1049/el:19881004

Zhou, W., Hua, Y., Huntington, M.D., Odom, T.W., 2012. Delocalized lattice plasmon resonances show dispersive quality factors. J. Phys. Chem. Lett. 3, 1381-1385. doi:10.1021/jz300318v

Zhou, W., Odom, T.W., 2011. Tunable subradiant lattice plasmons by out-of-plane dipolar interactions. Nat. Nanotechnol. 6, 423-427. doi:10.1038/nnano.2011.72 


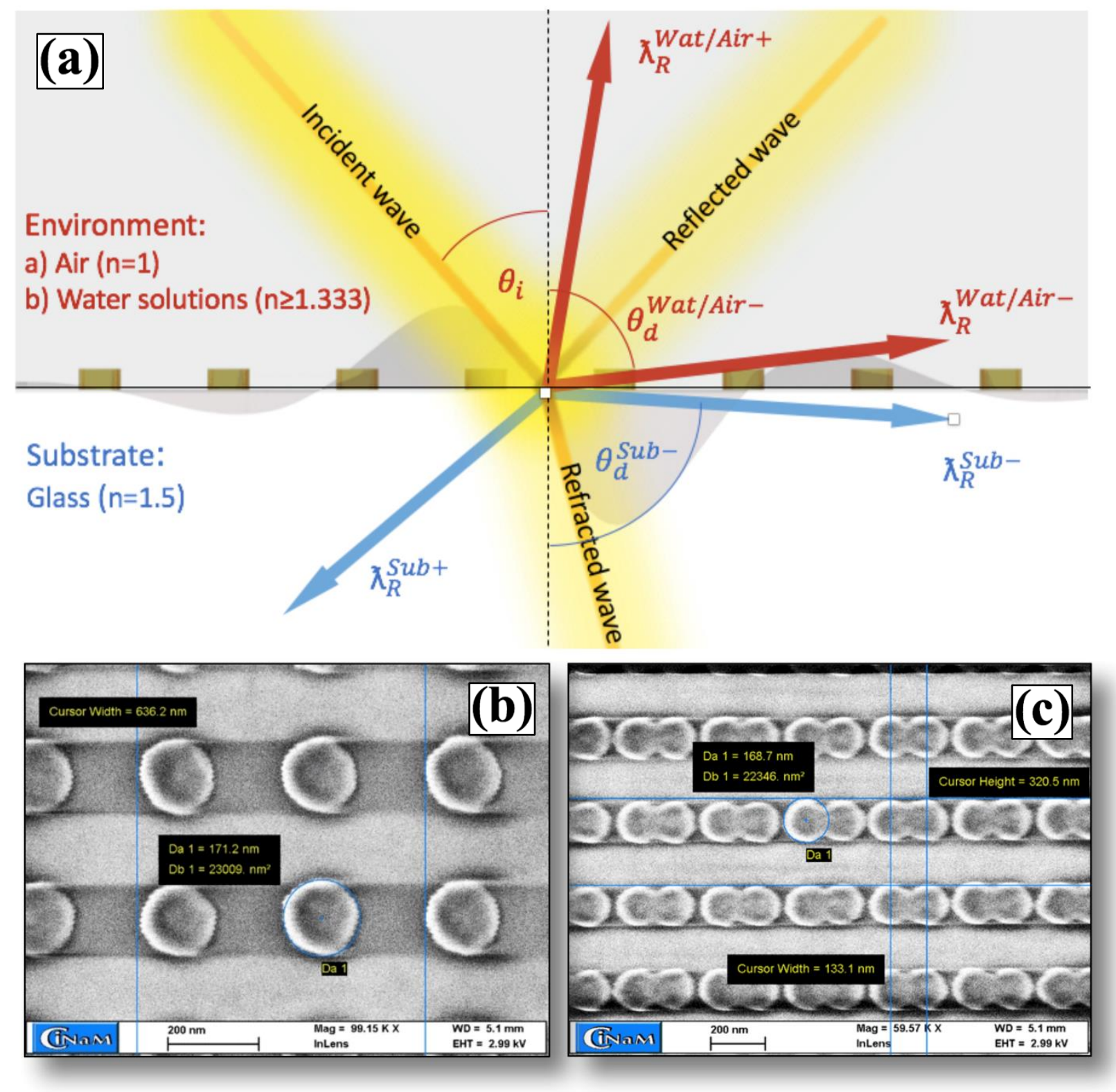

Fig. 1 (a) Schematics of excitation of diffraction-coupled plasmonic surface lattice resonances (PSLR) over a metal nanoparticle array. When metal nanoparticles are arranged in a periodic lattice, they may scatter light to produce diffracted waves. If one of the diffracted waves then propagates in the plane of the array, it may couple localized plasmon resonances associated with individual nanoparticles leading to the generation of PSLR with a very narrow lineshape. In the presence of a substrate, this phenomenon takes place at Rayleigh cut-off frequencies for the medium $\left(\lambda_{R}^{a i r}\right.$ or $\left.\lambda_{R}^{w a t}\right)$ and substrate $\left(\lambda_{R}^{s u b}\right)$; Typical Scanning electron Microscopy images of single (a) and double nanoparticle arrays used for the excitation of PSLRs. 

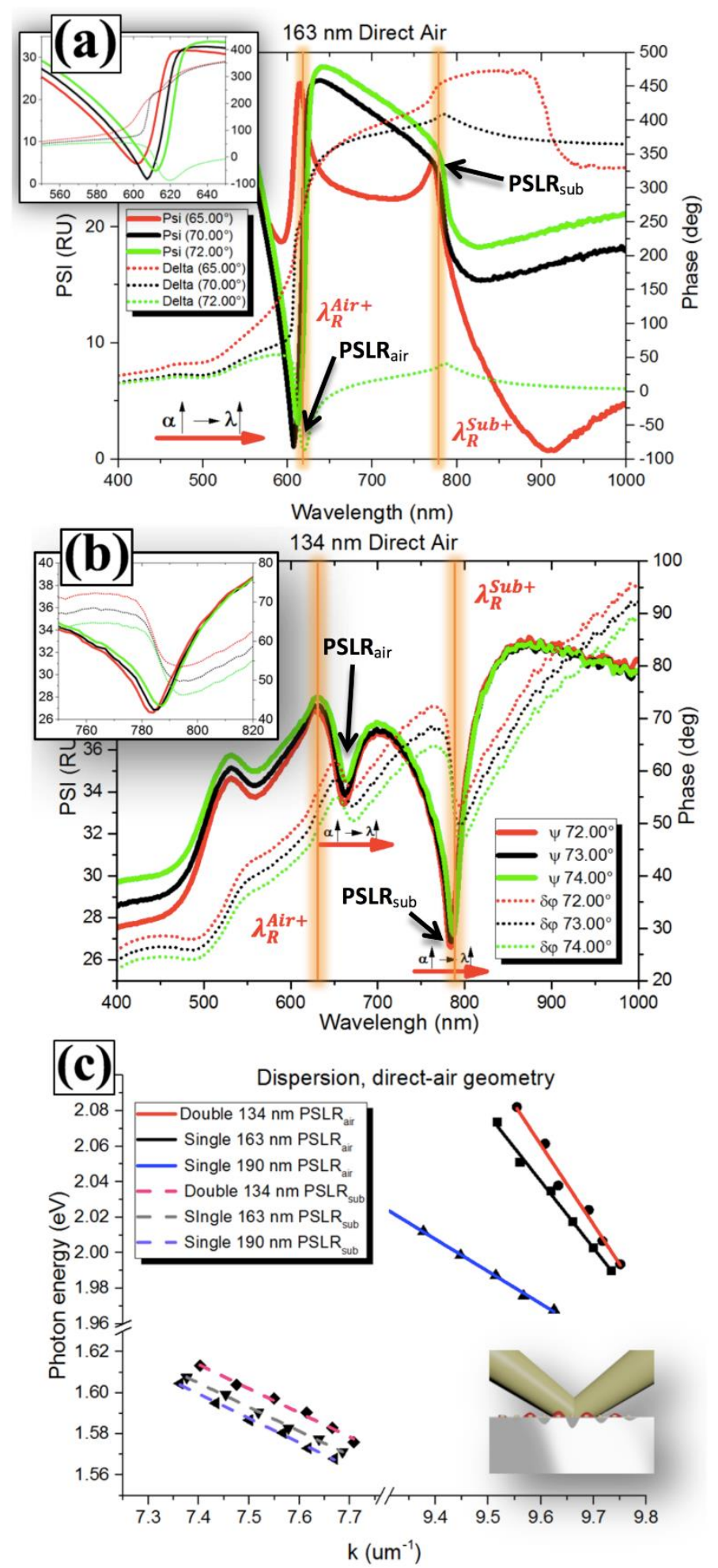

Fig. 2 Spectral dependencies of ellipsometric reflectivity $\Psi$ (solid) and phase $\Delta$ (dashed) for a 320-nm period arrays of glass-supported gold nanoparticles contacting with air medium under different angles of their illumination in direct geometry: (a) single 163-nm particle array; (b) double $134 \mathrm{~nm}$ particle array with the distance between the nanoparticles $50 \mathrm{~nm}$. Vertical yellow lines show positions of Rayleigh cut-off wavelengths associated with diffraction edges for air and substrate media ( $\lambda_{R}^{a i r}$ and $\lambda_{R}^{\text {sub }}$, respectively). The insets show some resonances at magnified scale. (c) Dispersion curves describing conditions for the generation of resonances (combinations of the resonance angle of incidence and wavelength) constructed on the basis of experimental data for air- and substrate-related modes PSLR air (solid) and PSLR $_{\text {sub }}$ (dashed) 

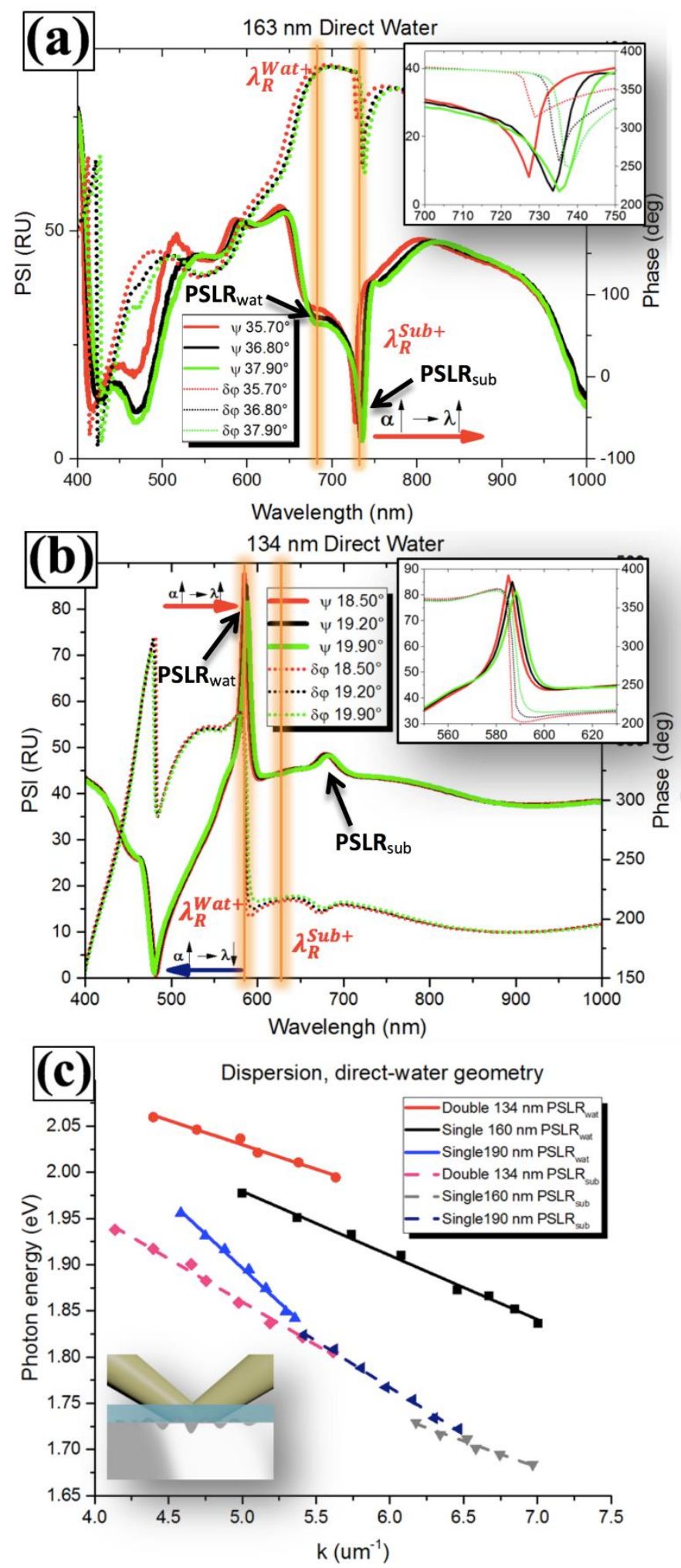

Fig. 3 Spectral dependencies of ellipsometric reflectivity $\Psi$ (solid) and phase $\Delta$ (dashed) for gold nanoparticle-based metamaterial arrays contacting with water medium under their illumination in direct geometry: (a) single 163-nm particle array; (b) double $134 \mathrm{~nm}$ particle array. (c) Dispersion curves for water- and substrate-related modes PSLR wat $_{\text {(solid) and }}$ PSLR $_{\text {sub }}$ (dashed) 

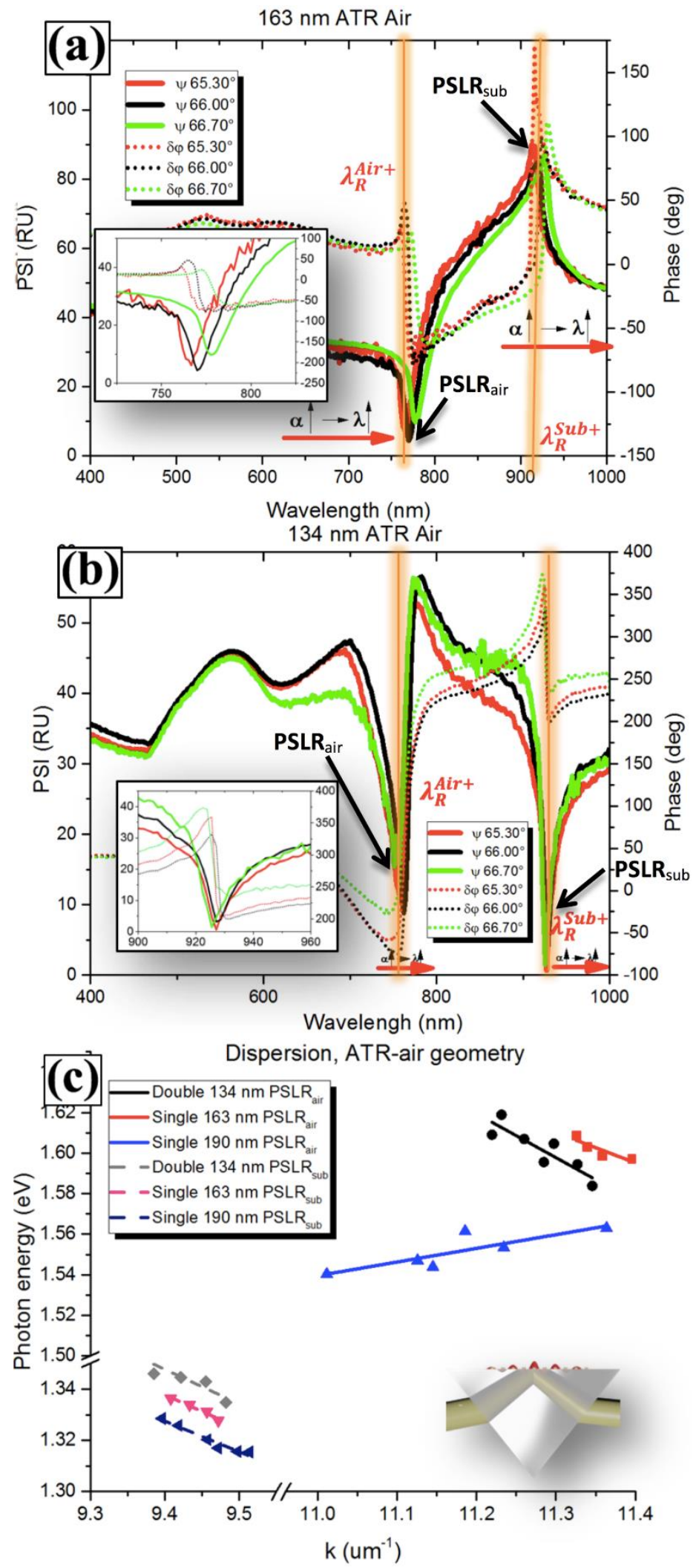

Fig. 4 Spectral dependencies of $\Psi$ (solid) and $\Delta$ (dashed) for gold nanoparticle-based metamaterial arrays contacting with air medium in Attenuated Total Reflection (ATR) geometry: (a) single $163 \mathrm{~nm}$ particle array; (b) double $134 \mathrm{~nm}$ particle array. (c) Dispersion curves for air and substrate-related modes PSLR air (solid) and PSLR sub $_{\text {(dashed) }}$ 

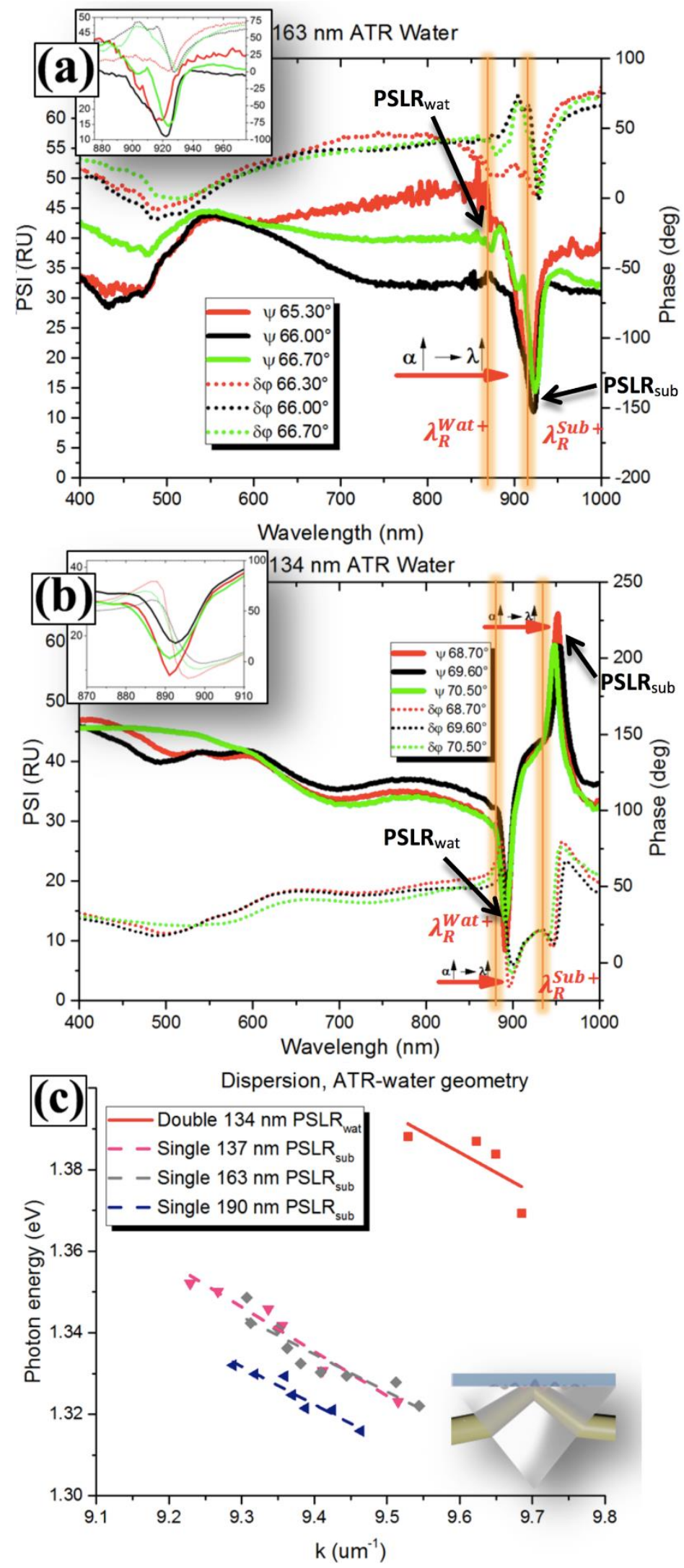

Fig. 5 Spectral dependencies of $\Psi$ (solid) and $\Delta$ (dashed) for gold nanoparticle-based metamaterial arrays contacting with water medium in Attenuated Total Reflection (ATR) geometry: (a) single 163-nm particle array; (b) double 134-nm particle array. (c) Dispersion curves for water and substrate-related modes PSLR air (solid) and PSLR sub $_{\text {(dashed) }}$ 

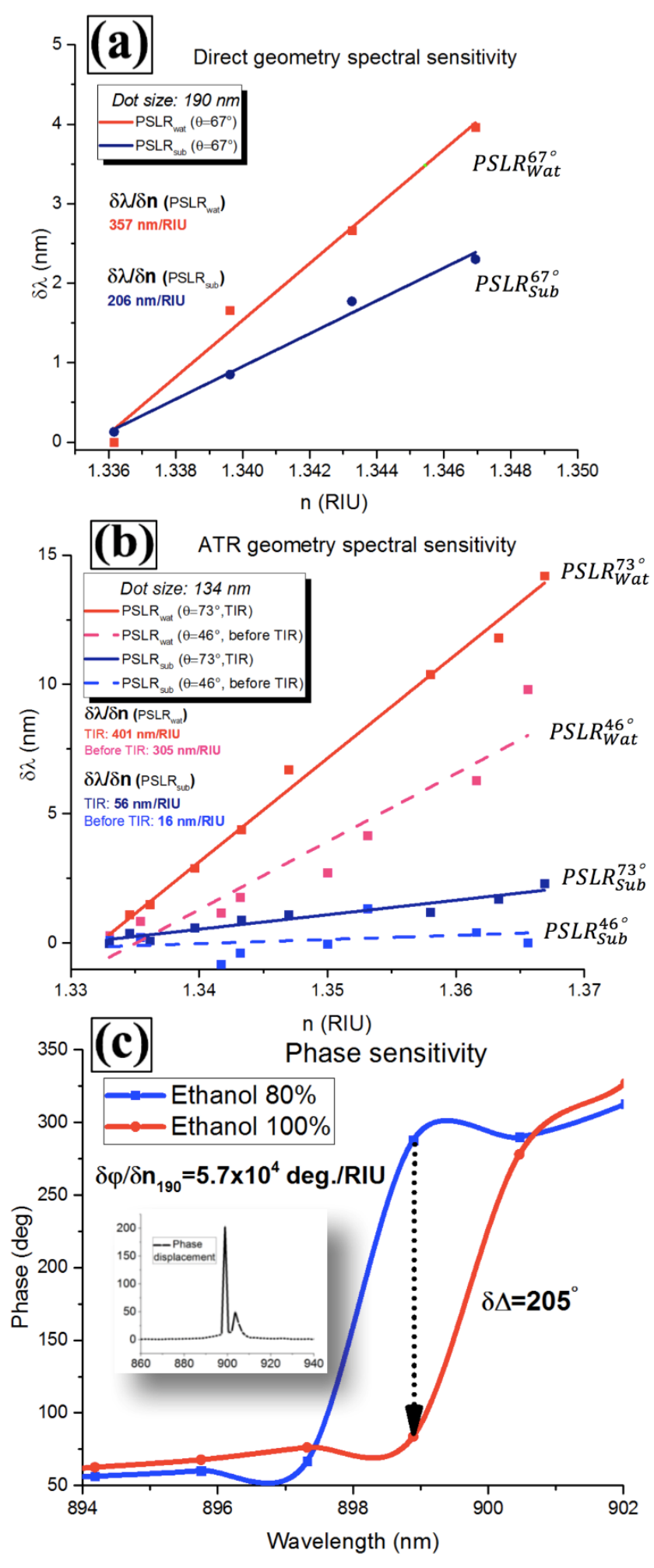

Fig. 6. Sensitivity measurements. (a) Direct geometry: Spectral response of medium PSLR wat $_{\text {(red) and PSLR }}$ sub (blue) modes excited over single $190 \mathrm{~nm}$ particle array (period $320 \mathrm{~nm}$ ) to RI changes conditioned by pumping of different ethanol/water mixtures through the flow cell. The angle of light incidence is 67 Deg.; (b) ATR geometry: Spectral response of medium PSLR wat $_{\text {(red) and PSLR }}$ sub (blue) modes excited over double $134 \mathrm{~nm}$ particle array (period $320 \mathrm{~nm}$ ) before (dashed) and after (solid) TIR to changes of RI. The angle of light incidence is 67 Deg.; (c) Typical spectral dependences for $\Delta$ under the change of RI by $4.4 \cdot 10^{-3}$ RIU. The inset shows the difference phase signal $\delta \Delta$ for phase curves indicated in the figure. 